\title{
Programmable Logic Controllers and Data Traffic Handling Solutions
}

\section{Dr. David Border, Bowling Green State University}

David A. Border, Ph.D., holds a principle research interest in electronic information systems. This field includes digital communication and networking and intelligent networked devices. His work includes wireless sensor networks. Prior research included work on signal bandwidth compression and signal specific data encoding techniques. His technology application interest includes networked systems. Typical teaching duties include junior- and senior-level courses in the Electronics and Computer Engineering Technology (ECET) program. Within this course set are the curriculum's networking and communication courses. As is true with his ECET faculty colleagues, Border supports the program with teaching assignments, as needed, in freshman- and sophomore-level courses offerings. Examples of these include the sophomore level electric circuits and digital electronics courses. Border teaches a digital communication graduate course within a Ph.D. Consortium Technology Management program, as well as other graduate level courses at BGSU.

Border served as interim department chair of the Engineering Technologies department. He served as chair of the university Faculty Senate curriculum and academic affairs committee. He is chair of the University Faculty Senate. 


\section{Abstract}

Today's manufacturing industry depends on Programmable Logic Controllers (PLCs). Throughout the industry, engineers are using PLCs to collect and keep track of vast quantities of data. There are many different ways that industry uses to accomplish the task of retrieving and reporting useful data from PLCs. End-to-end transfers use large application tools, others use open software, and yet others use proprietary solutions. Incorporating laboratory instruction on the handling of such transfers in an educational laboratory and making such capabilities available for senior project work can be very useful for graduates. This project considers two very different strategies for monitoring and collecting PLC data for student instruction.

The first strategy is the use of free demonstration software to implement an industry open standard solution, OPC UA servers that work with a broad number of devices. Considered are three OPC UA server vendors: Ignition Automation, Kepware, and Matrikon.

The second strategy is the use of a proprietary application solution that provides data monitoring or operator interactions enabled through its proprietary communication standard. Two separate sites, the classroom laboratory and an industry site hosted this phase of the work. In each case, the data traffic source is a PLC. The data traffic destination is a Windows PC. The PLC and PC share a LAN connection and all data traffic is over Ethernet. Both strategies work well, yet the advantages of the open architecture strategy, using demonstration software, is judged to be the most favorable solution for the classroom and laboratory. 


\section{Introduction}

In a recent ASEE conference paper [1], its author presented the case for broader instructional goals in introductory Programmable Logic Controller (PLC) courses. He cited the need to include system communication skills to support supervisory control and data acquisition tasks, compelling elements in many curricula. Useful laboratory configuration details are in the body of work. In particular, the PLCs all had Ethernet physical connections; Object Linking and Embedding (OLE) for Process Control (OPC) was the standard network data communication method over Ethernet. The author also cited the high costs of OPC-based system servers and clients. Therefore, free demonstration-based OPC software was selected for use. This work focused on one vendor's OPC Server/Client applications, Matrikon Technologies.

In an earlier ASEE conference paper [2], its authors presented a case for investigating a similar subject matter, handling laboratory data in an OPC-based networked system in the context of a senior level course on industrial networks. The paper detailed the hardware/software collection of process variables from a Controller Area Network (CAN-bus) onto an OPC server. The lab shown used Kepware Technologies demonstration OPC Server/Client software. Although the lab employed data collected on a CAN-bus, it seems reasonable that PLC-based data could have been used in the lab instead since PLC knowledge was a course prerequisite and PLCs were elements of the course.

Based on these ASEE proceeding's papers, there is agreement that engineering technology labs must deliver new content in their curricula on the subject of OPC-based networks. Further, there is agreement that students who are learning or have learned PLC fundamentals should know of those systems, as the PLC is a typical system data node. It is the goal of the following sections to describe work that supports this assertion, by (1) studying demonstration-based OPC software and (2) investigating licensed, proprietary, industrial network software. A PLC will be present in all the working testbeds since the factory PLC is available in the teaching laboratory. Testbeds were created both at our laboratory and at a local industry site. Use of the industry site allowed testing of some proprietary software that is not present in our class laboratory.

\section{Site Hardware and Licensed Software}

The hardware used for the work varied somewhat by location. At the industry site, RS 500 software and MicroLogix 1100 PLCs were used. In our class laboratory, CompactLogix PLCs are used. In each location, Ethernet switches carried the information to and from devices on a Local Area Network (LAN). In each location, Windows-based PCs were used. Because the commodity nature of computing has caused a relative abundance of reliable PCs at reasonable costs, the amount of computer system memory, hard disk drive capacity, computer clock speed and the count of CPU cores available were not issues of concern.

Licensed software varied by location. For PLC programming, the industry site makes use of licensed Rockwell Automation/Allen Bradley software supporting its RSLogix 500 software; the class laboratory uses licensed Rockwell Automation Studio 5000. For Human Machine Interfacing, the industry site makes use of licensed IDEC [3]Wind Touchscreen Operator Interface (WindO/I) software; the class laboratory uses licensed Rockwell Automation FactoryTalk®View software.

The key to Rockwell Automation task connectivity is its licensed product, RSLinx. For purposes of this work, task connectivity means Excel Spreadsheet and FactoryTalk ${ }^{\circledR}$ View communication with Allen Bradley PLCs. RSLinx is a useful application, and it supports active-network browsing of 
Rockwell Automation industrial communication connections, including those based on Ethernet. Rockwell does offer a free version of RSLinx called RSLinx Lite. The lite version does not support OPC or Excel connectivity. Therefore, RSLinx Lite was not appropriate for this work.

\section{Importance of Object Linking and Embedding for Process Control (OPC)}

OPC Unified Architecture (OPC UA) [4, 5], by design, is intended as a "service-rich" standard [6, 7, 8, 9]; thus for end-users, it accommodates the wide variety of features (facets) anticipated by industry. By design, it empowers vendors to make that content possible. It is platform-independent and is messagebased. By design, it is mappable onto existing communication protocols using the Client/Server model. Importantly, since it adopts the Client/Server model, the classic formulation of assigning system responsibilities by layer, as (1) User Application, (2) Application Program Interface (API), (3) Communication Stack and (4) Physical Layer, can be employed. Using conventional approaches ensures adoption by developers.

For those familiar with Network Layer implementation models, the following visualization, Figure 1, should look familiar. It shows the Server/Client OPC Network Model philosophy used with OPC UA and has its inspiration from the ISO OSI reference protocol stack model. It is an example of good practices within the OPC UA standard.

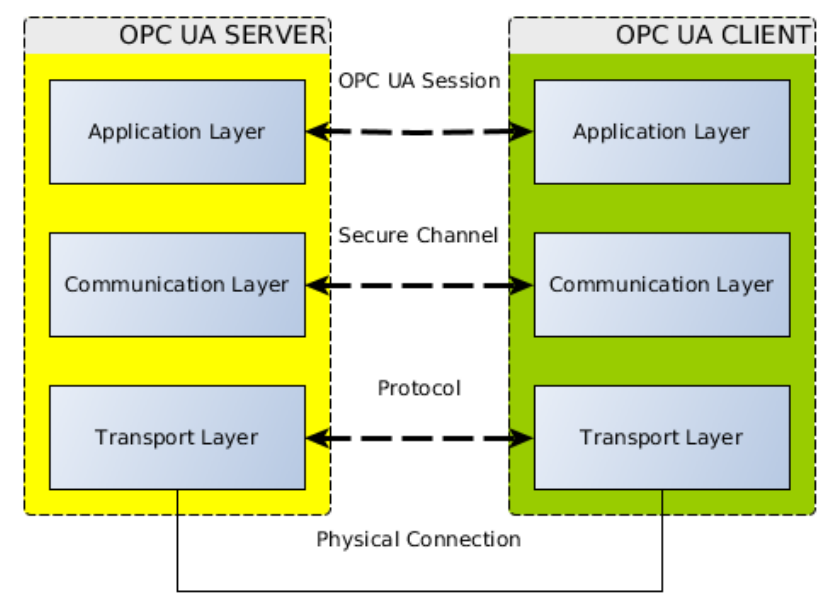

Figure 1. OPC UA Network Model

The OPC Foundation currently cites three software development environments as being used for OPC UA products. They are Java, .Net and $\mathrm{C} / \mathrm{C}++$. Additionally, volunteers have contributed a Pythonbased beta OPC-UA client and server library. Some vendors now market software OPC-UA software development kits (SDK), these include Matrikon, Prosys, Unified Automation, and Softing.

\section{OPC UA Server and Client Candidates}

Based on the earlier cited ASEE proceedings, two demonstration candidate OPC UA Servers were procured for this project, Kepware OPC UA Server and Matrikon OPC UA Server. A third demonstration OPC UA Server candidate, from Inductive Automation [10], was also obtained. Table 1 summarizes the basic installation information for each vendor. 


\begin{tabular}{|l|l|l|}
\hline Vendor & Target OS & License Period \\
\hline Matrikon & Windows & 30-days \\
\hline Kepware & Windows & $\begin{array}{l}\text { 2-hour total clock-time per session, } \\
\text { reactivation “click” between sessions. }\end{array}$ \\
\hline Inductive Automation & $\begin{array}{l}\text { Windows, Linux, } \\
\text { MacOSX }\end{array}$ & $\begin{array}{l}\text { 2-hour total clock-time per session, } \\
\text { reactivation “click” between sessions. }\end{array}$ \\
\hline
\end{tabular}

Table 1. Basic Installation Information on Demonstration OPC UA Servers

The Inductive Automation product is named "Ignition.” It is a Java-based application, and since Java is on many platforms, including Linux, it is not surprising that Ignition is offered for Linux. Matrikon, while supporting SDK for both Linux and Windows developers, chose to target their OPC UA Server demo to Windows-only platforms. Kepware is a "Windows-only" OPC UA developer, and its demo runs on Windows-only platforms. Each of the server packages provides a set of industry functionalities through a Graphical User Interface based tools; Table 2, lists the minimum common functions/tasks of all the vendor packages.

\begin{tabular}{|l|l|}
\hline \multicolumn{1}{|c|}{ Function } \\
\hline Connection to OPC Data Sources (Devices) \\
\hline Read/Write by Tag Name \\
\hline Accept Connection from OPC Clients \\
\hline
\end{tabular}

Table 2. Common OPC Functions Shared by All Three Vendors.

Each vendor markets a set of "device drivers," or just "devices," with their server. The Inductive Automation server is focused on supporting PLC connections. It also supports TCP-based MOD-Bus connections. If a PLC course is being taught using Siemens or Allen Bradley equipment, the Inductive Automation product should be useful. The Matrikon server and Kepware server each support a large number of PLCs; unlike Inductive Automation, they also support a large number of non-PLC devices.

The OPC UA Clients tested were in two categories, (1) clients bundled into the OPC UA Server distribution and (2) license and cost-free third-party vendor clients. Bundling the clients is a nearmandatory requirement when selling or demonstrating a server application. However, since the OPC architecture is now "open" and standards are in place, free third-party client software exists, too. Table 3 lists the OPC clients in this work. 


\begin{tabular}{|c|c|c|c|}
\hline Marketed & Vendor Name/Product Name & Target OS & Demo-License Period \\
\hline \multirow[t]{3}{*}{$\begin{array}{l}\text { Bundled with } \\
\text { Server }\end{array}$} & $\begin{array}{l}\text { Matrikon } \\
\text { Product: Matrikon Explorer }\end{array}$ & Windows & 30-days \\
\hline & $\begin{array}{l}\text { Kepware } \\
\text { Product: OPC Quick Client }\end{array}$ & Windows & $\begin{array}{l}\text { 2-hour total clock-time } \\
\text { per session, reactivation } \\
\text { “click” between sessions }\end{array}$ \\
\hline & $\begin{array}{l}\text { Inductive Automation } \\
\text { Product: Ignition Designer }\end{array}$ & $\begin{array}{l}\text { Windows, } \\
\text { Linux, } \\
\text { Mac OSX }\end{array}$ & $\begin{array}{l}\text { 2-hour total clock-time } \\
\text { per session, reactivation } \\
\text { "click” between sessions }\end{array}$ \\
\hline \multirow[t]{2}{*}{ Third party } & $\begin{array}{l}\text { Unified Automation } \\
\text { Product: UA Expert }\end{array}$ & $\begin{array}{l}\text { Windows, } \\
\text { Linux }\end{array}$ & Standard version (free) \\
\hline & $\begin{array}{l}\text { Prosys } \\
\text { Product: OPC UA Client }\end{array}$ & $\begin{array}{l}\text { Windows, } \\
\text { Linux }\end{array}$ & Standard version (free) \\
\hline
\end{tabular}

Table 3. Basic Installation Information on OPC UA Clients Procured

\section{The OPC UA Server Test}

For testing a small dedicated local area network (LAN) was used. A Cisco 1900 series switch provided network connectivity. An Allen Bradley CompactLogix Controller, with a power module and a six input/four output digital I/O module, connected directly to the switch. A PC-computer also connected directly to the switch (see Figure 2).

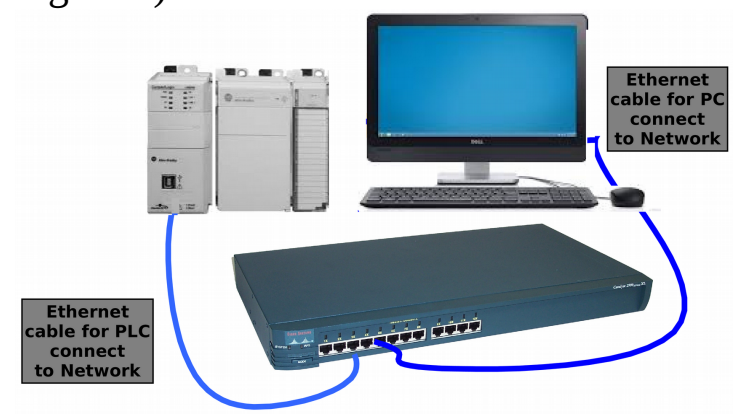

Figure 2. Hardware Testbed

Before being used for this project, the PC chosen had no PLC communication or related application software installed. This insured that there were no lingering software modules in the OS, libraries, or file system that could adversely affect testing. In a separate operation, an exercise ladder logic program was coded and downloaded into the CompactLogix PLC. The exercise consisted of a Timer block, Math block, Move block, timer reset, and two boolean (examine on, examine off) instructions. Central to the logic was the establishment of a timer value moving from 0 to 15000 milliseconds, followed by a timer reset and repeat. The Math block computed an integer "seconds" value. The first boolean controlled the start/stop of the timer and the second boolean controlled a conditional copy of the integer "seconds" value to the hardware digital output. Therefore, four entities existed in the PLC program 
memory, (1) Timer Structure (millisecond units), (2) Integer Timer in seconds, (3) Boolean "System" Start/Stop and (4) Boolean "Copy to Output Coils" Enable/Disable.

Each project OPC UA Server was installed and tested. Figure 3 provides a flowchart showing the general tasks common to the setup of the servers.

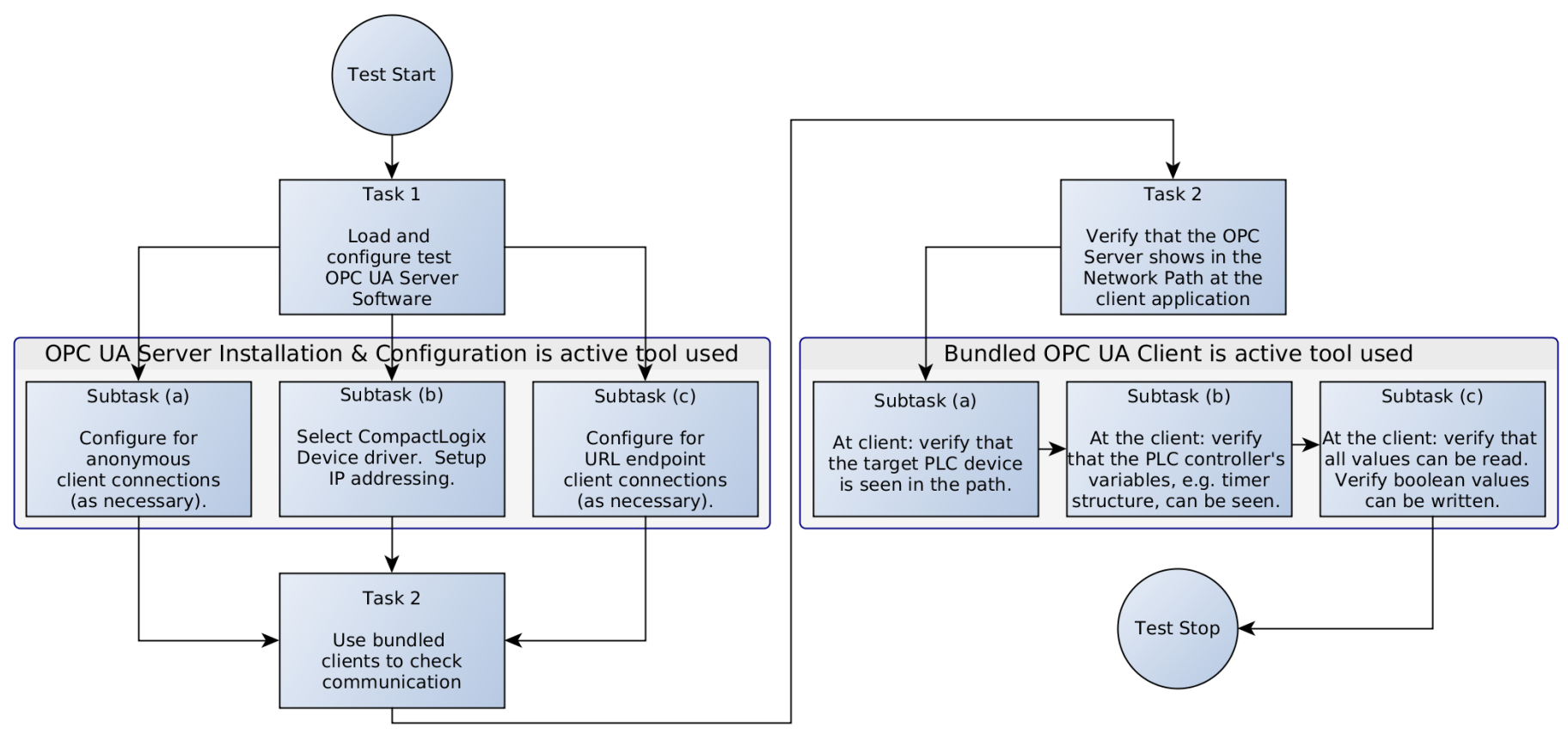

Figure 3. Installation and Verification of an OPC UA Server

The installation's objective measure of success for a given vendor was whether its OPC UA client and third-party clients, when connected, could read the two timer tags and read/write the two boolean tags. The installation's subjective measures of success were the consistent presence of application "userfriendliness" and "ease of operation.” All three server vendors met these goals. All four tags were readable with the boolean tags being writeable. The "seconds" timer was particularly helpful since it acted as like a "heartbeat" signal. Figure 4 shows a case of a "client to server" communication. It shows the "Prosys UA Client" successfully browsing the tags within the PLC via the Inductive Automation OPC UA server. Also shown in the figure is the value of the PLC heartbeat timer, its value is " 2. ." The tag name " $\mathrm{t}$ " is reversed highlighted. 


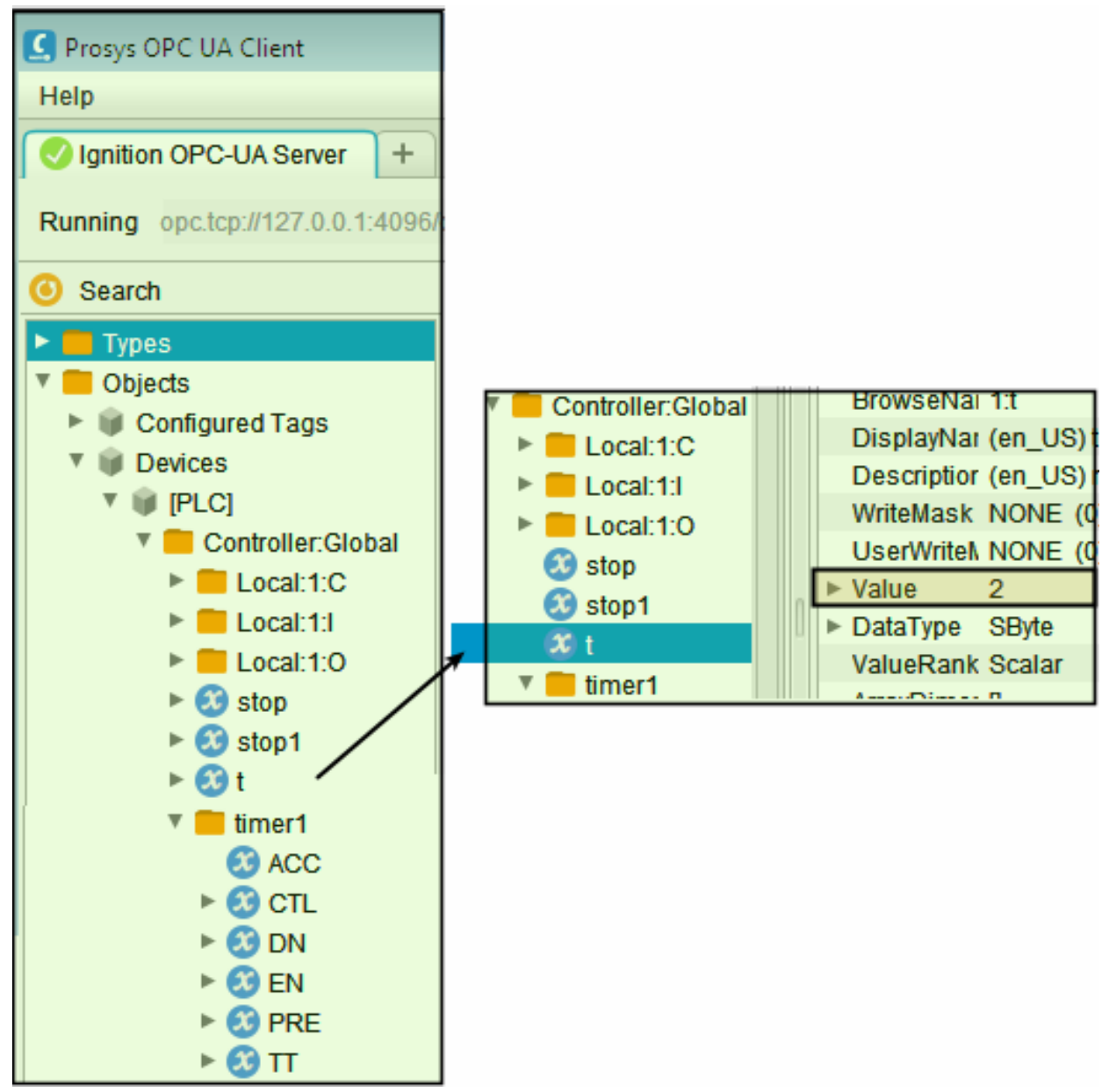

Figure 4. Client UA Communicates to PLC through Server

The subjective goals were met by each vendor. Here are observations, (1) Matrikon's set up process seemed logical and thorough (2) Kepware's operator interface seemed quite useful and (3) Inductive Automation's installation uses an HTML/web interface that was very attractive and useful. Therefore, based on the measures, all three OPC UA server packages were judged effective.

\section{Notable Observations Concerning the Operation of the OPC UA Servers}

Successful completion of the installations led to further tasks. Unlike the previous section, these tasks were not meant to create formal cross comparisons among the three vendors. The first task involved work with a Linux OS. Since the OPC UA standard is crafted to promote "multi-platform" development and deployment of servers, it was decided to install the Inductive Automation Linux OPC UA Server on an Ubuntu Linux distribution.

The configuration and administration of the Inductive Automation Linux-based software were identical to the Window's version. Features and operation of its embedded "client" Ignition Designer were identical. This checkout phase allowed for the investigation of the "designer" features in Ignition. One such feature is its native Human Machine Interface (HMI) capabilities. These capabilities are not in the Matrikon or Kepware products. With some knowledge of how to work with designers, such as with GUI application designers, it is possible for a user to build a small Ignition-based HMI solution without much difficulty. Figure 5 shows an Ignition-based design working with tags in the simple PLC ladder logic program discussed in Section V. 


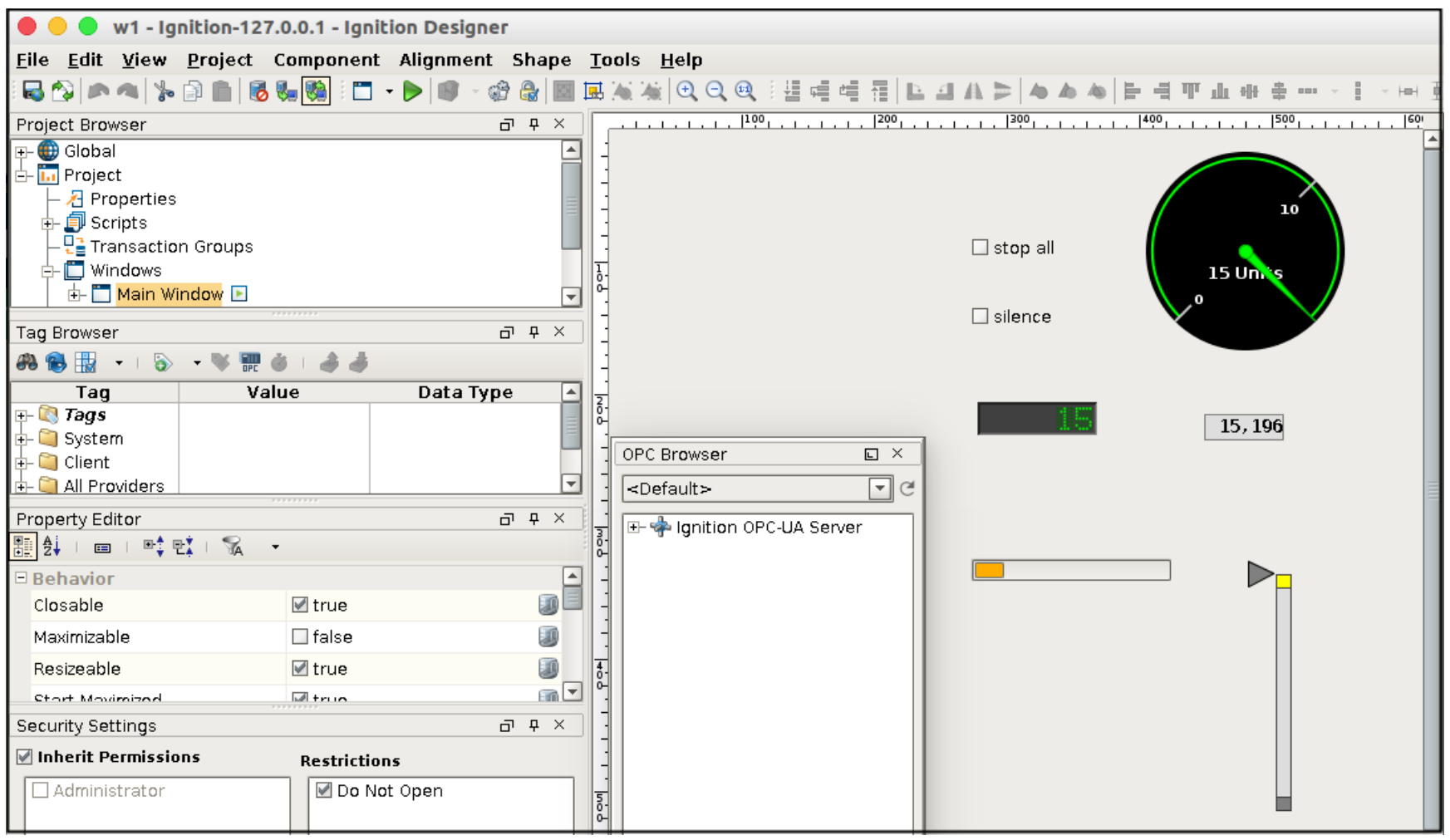

Figure 5. An Ignition Designer based HMI

The integer "seconds" timer, "t" are from the browse shown in Figure 4. The HMI uses five different, but redundant, “components,” (1) Vertical Analog Moving Indicator, (2) Horizontal Progress Bar, (3) Meter, (4) Numeric Label and (4) LED Display to display the integer time value. The two booleans are reported and activated using the "checkbox" component.

The Matrikon \& Kepware embedded “abilities” are considered limited, therefore a 30-day trial Windows-based application product from “Open Automation Software,” [11] was loaded. It is a product whose features include browsing OPC UA servers, data logging, alarming, and recipe handling. Basic “*.cvs” data logs were created easily from both the Matrikon and Kepware servers. A sample data log file is shown in Figure 6. It shows the timestamp, the boolean "STOP1" tag value and the integer " $t$ " tag value.

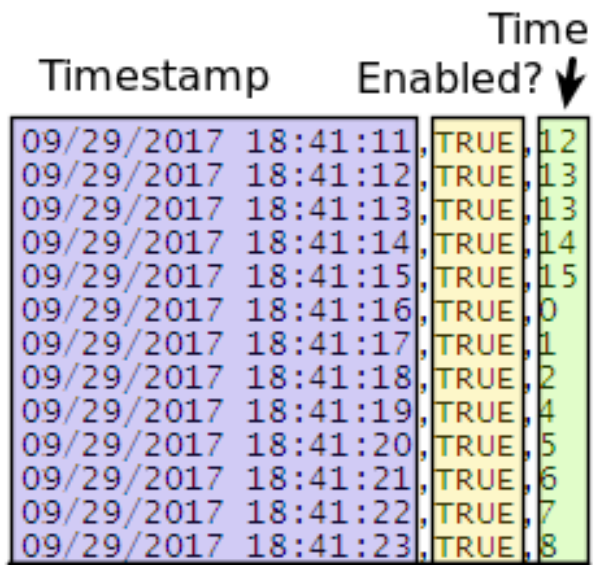

Figure 6. Sample Log File using Open Automation Software 
The Open Automation Software (OAS) package also includes an Excel OPC tool, for use with Excel's Real Time Data (RTD) function. The RTD function displays OPC-based real-time data. In a somewhat lengthy string based argument, the RTD function learns the name of the data source (server) and the name of the data to be retrieved. The OAS tool enables (1) the selection of the data OPC UA server (data source), and (2) OPC UA tag browsing to select the data source. The OAS tool builds the entire RTD function string. This function string is then inserted into an Excel cell manually (copy-and-paste). OAS Excel abilities were testing using the OPC-based boolean "stop1" and the integer " $\mathrm{t}$ " (also used in Figure 6). Figure 7 shows the Excel results. Excel Real-Time functions will be discussed further in Section VIII.

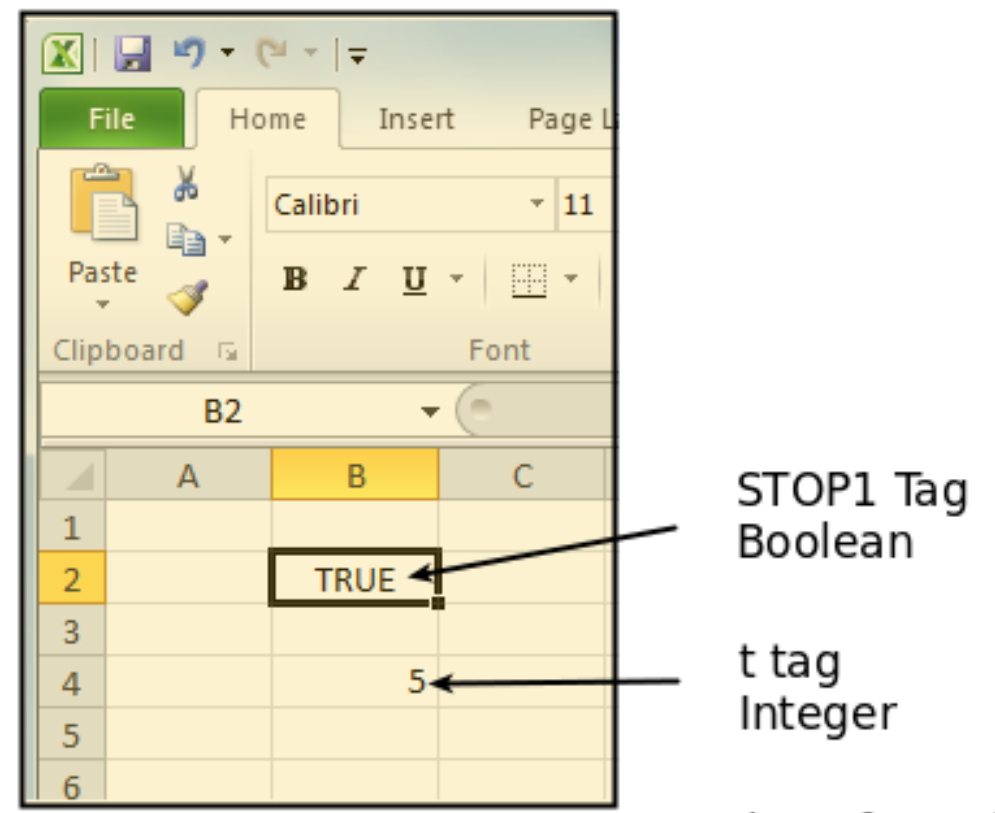

STOP1 Tag

Boolean

t tag

Integer

Portion of Excel Cell using RTD function

=RTD("opcexcel"," "\\127.0.0.1\DirectOPC:Kepware.KEPServerEX.V6"

Figure 7. Open Automation Software’s Excel Real-Time Data Function

While Matrikon currently distributes its OPC UA server demonstration software only for Windowsbased PCs, they provide a small, free, Linux-based OPC UA Client application. What is notable is that it is a command line-based, text only, client. It was downloaded and tested successfully. Appendix I reports the successful test results.

VII. Licensed Industrial Network Applications - Deployed in the Classroom Lab

The next phase considered communication through a licensed application to a PLC in the class laboratory. Work began with the licensed Rockwell FactoryTalk ${ }^{\circledR}$ View product. FactoryTalk ${ }^{\circledR}$ View can attach to devices (e.g., PLCs) through official Rockwell Automation third-party OPC partners. However, in Rockwell client sites RSLinx is most generally used for communication services. This product is bundled into many Rockwell products, including the Rockwell Studio 5000 application software. Studio 5000 is a licensed product in the lab. RSLinx provides OPC communication services to FactoryTalk®View. 
The general system capabilities of FactoryTalk®View is a union of features from the Open Automation Software application and the Ignition Designer application. This work investigated the HMI FactoryTalk®View capabilities, intentionally comparable to work seen earlier in Figure 5.

Just as the demonstration OPC UA servers required setting the up the device (subtask (b) in Figure 3), the FactoryTalk ${ }^{\circledR}$ View device must also be set up. RSLinx is invoked in the configuration task section of FactoryTalk®View. It returns the IP address of the Allen Bradley CompactLogix PLC (the "device"). The setup steps assure full PLC to HMI communication association.

Once the device is active, the design of a FactoryTalk ${ }^{\circledR}$ View HMI solution proceeds to the next phase, tag selection, and component layout. As with Ignition Designer tag browsing is present in FactoryTalk ${ }^{\circledR V i e w}$ and design components can be dropped onto the HMI design workspace. Design component properties are present and editable. The component communication property is edited to point to the appropriate PLC tag names. Once the design space is populated with edited components, the Panel Display running FactoryTalk ${ }^{\circledR V i e w}$ is functional. Figure 8 shows a "running” FactoryTalk®View HMI.

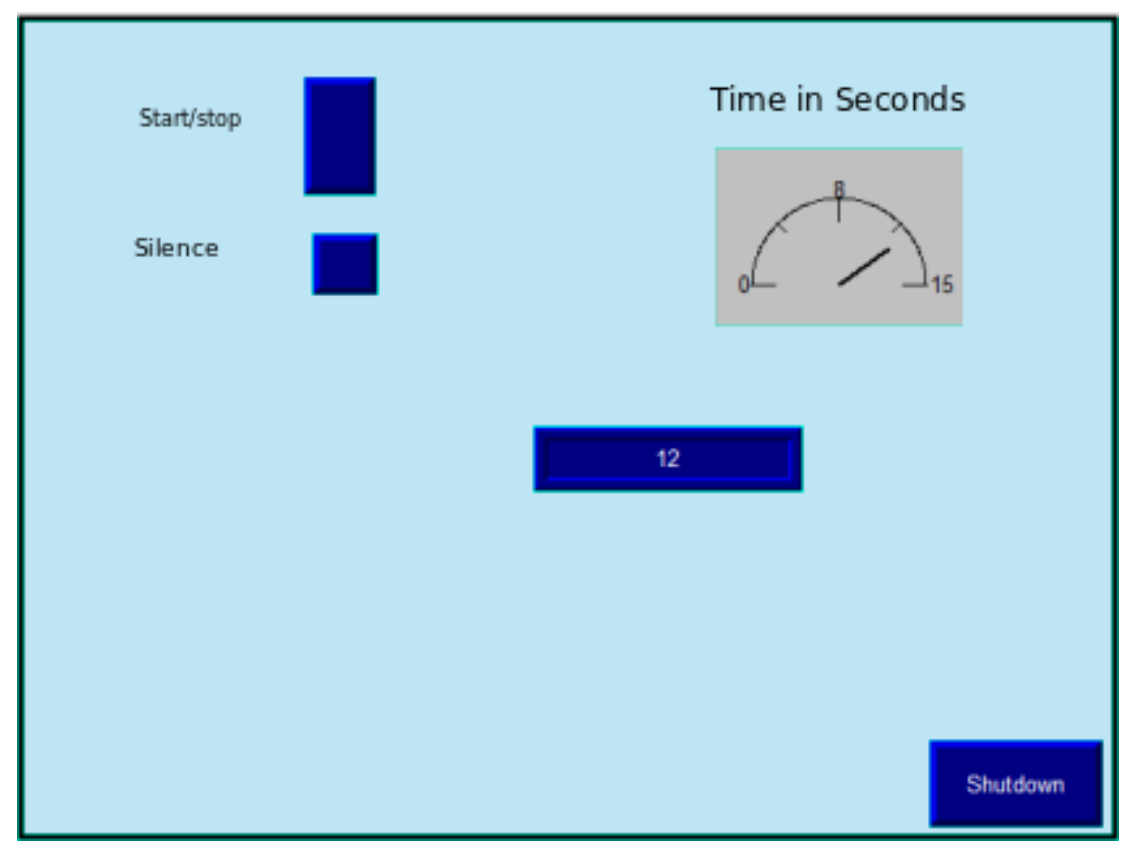

Figure 8. FactoryTalk ${ }^{\circledR V i e w ~ H M I ~}$

\section{Licensed Industrial Network Applications - Deployed at an Industry Site}

As stated in Section II, Rockwell's RSLogix 500 was the licensed PLC application software in use at the industry site. Also the industry site used IDEC's licensed Operator Panel (HMI) WindO/I application software for its production line automation. In the production line segment for the subject of this work, an Allen Bradley MicroLogix 1100 PLC was used. Thus it was not possible to choose the data handling application code or the PLCs used or to program the PLCs. There was an opportunity to examine the configuration of an existing HMI panel. Functionally, their HMI "parts bin” in use was divided into groups: Buttons, Indicators, and Counters/Timers/Indicators as shown in Figure 9. 

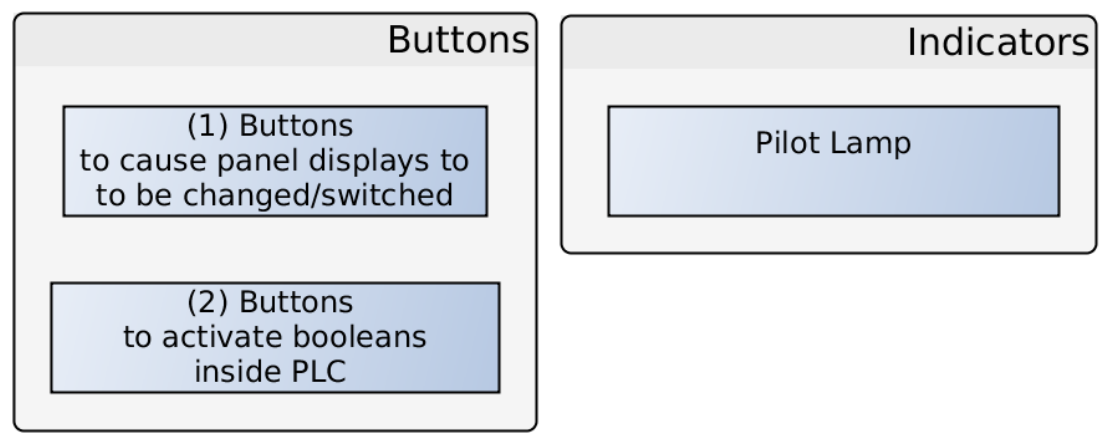

Timers/Counters/Integer

A Numeric Display

Figure 9. HMI Display Components used in the Industry Panel

The initial panel page consisted of 5 buttons, 24 pilot lamps, and 7 numeric displays components. Subsequent panels from the same project used as many as 23 pilot lamps. There were a total of five panels used in the industry HMI. The WindO/I panel configuration steps force the designer to specify all component properties. All tag addressing is done at the bit level using SLC-500 style addresses. The communication set up dialog allows specification of the PLC's IP address and Port number for a project. The WindO/I driver makes the direct IP network connection from itself to the MicroLogic 1100 PLC. Figure 10 shows an HMI panel.

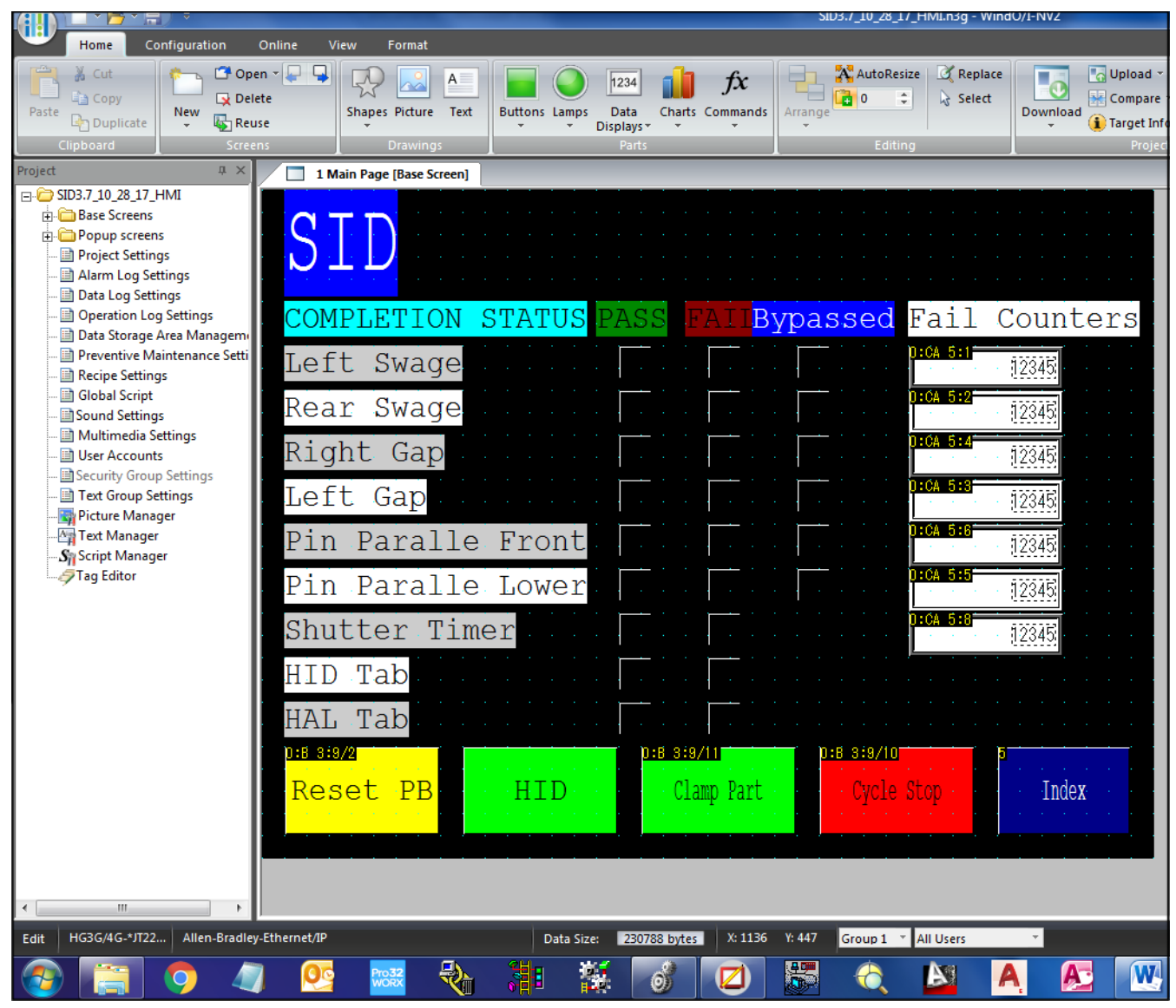

Figure 10. A WindO/I Operator Panel 
There was no opportunity to make changes to the IDEC WindO/I HMI. However, there was an opportunity to use the Excel Dynamic Data Exchange (DDE) based capabilities to build a real-time data monitoring display. The DDE protocol is for inter-program messaging and is native to Windows. The Rockwell Automation communication program, RSLinx, is DDE capable. If an Excel cell invokes a DDE compliant program, RSLinx in this case, a data channel is opened and data populates into the cell. Figure 11 shows a sample Excel cell. RSLinx calculates the cell address, PLC_01 B3:0,L1,C1. Figure 12 illustrates the general steps using RSLinx to (1) create the remote device, known in RSLinx as the "topic," and (2) create the "Data Table Address."

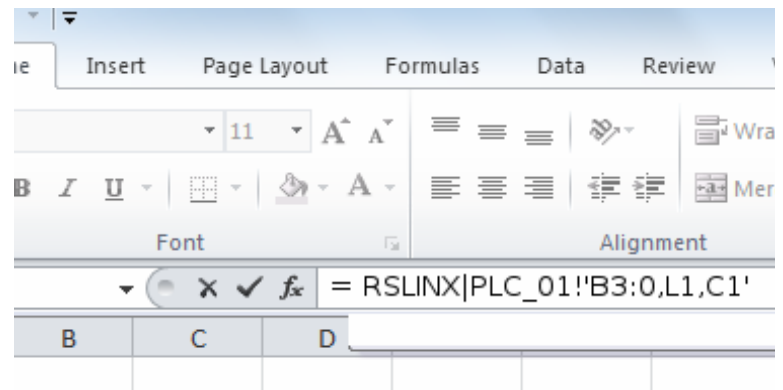

Figure 11. Sample Excel Cell Content
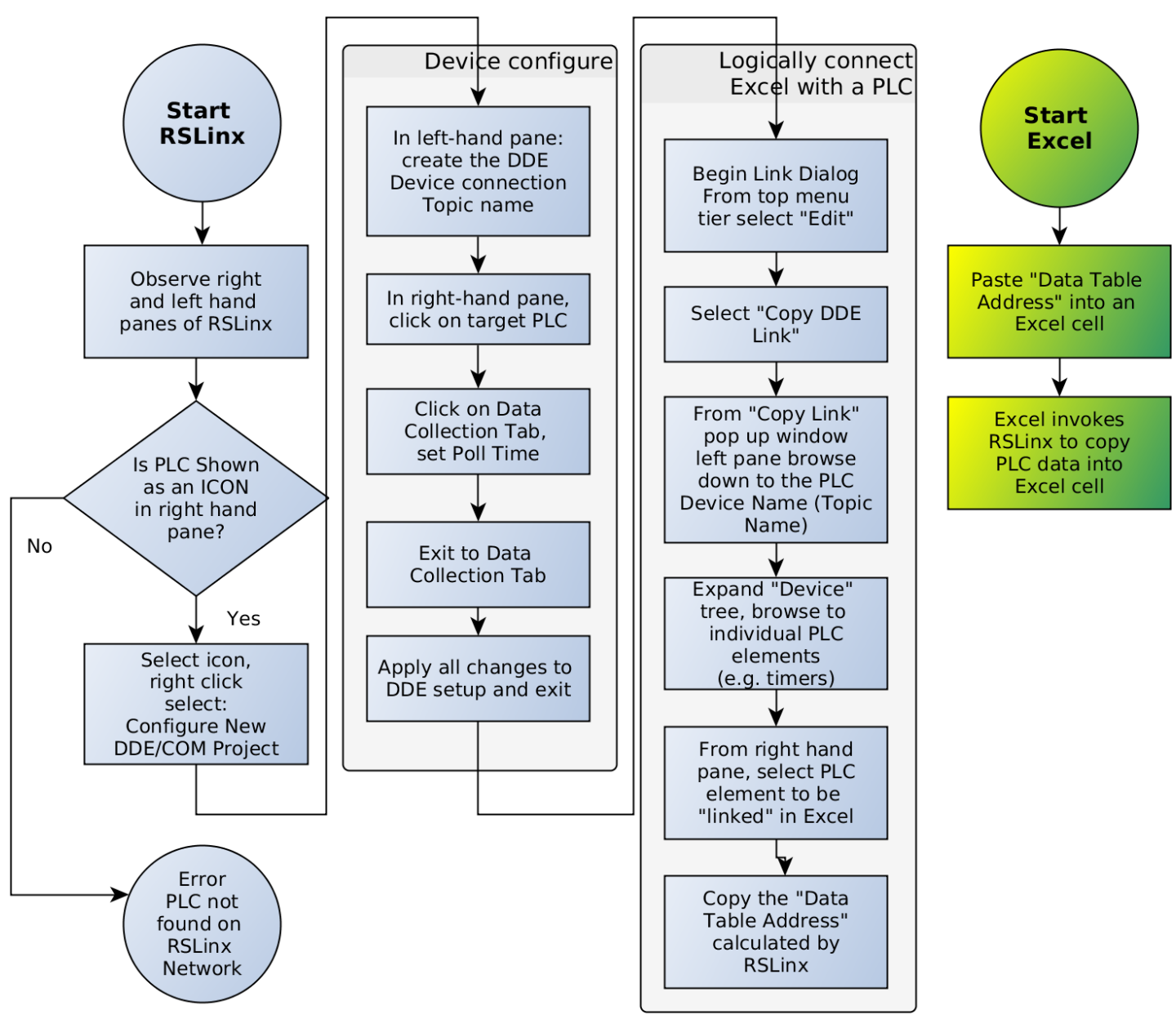

Figure 12. RSLinx “Data Table Address” Calculation for Excel 
As a documentation exercise, all points of the MicroLogix 1100 PLC were organized into a spreadsheet by their hardware slot number. In text strings, these values were recorded: (1) plant tag name, (2) SLC 500 address, and (3) RSLinx "Data Table Address." Using this text reference, the Excel Real-Time Data Report sheet was fully assembled. The correctness of the Excel to PLC addressing was confirmed on a point by point basis during intervals when the plant line was not running production. The industry site did not allow the Excel program to be run during production. The constructed Excel spreadsheet is shown in Figure 13.

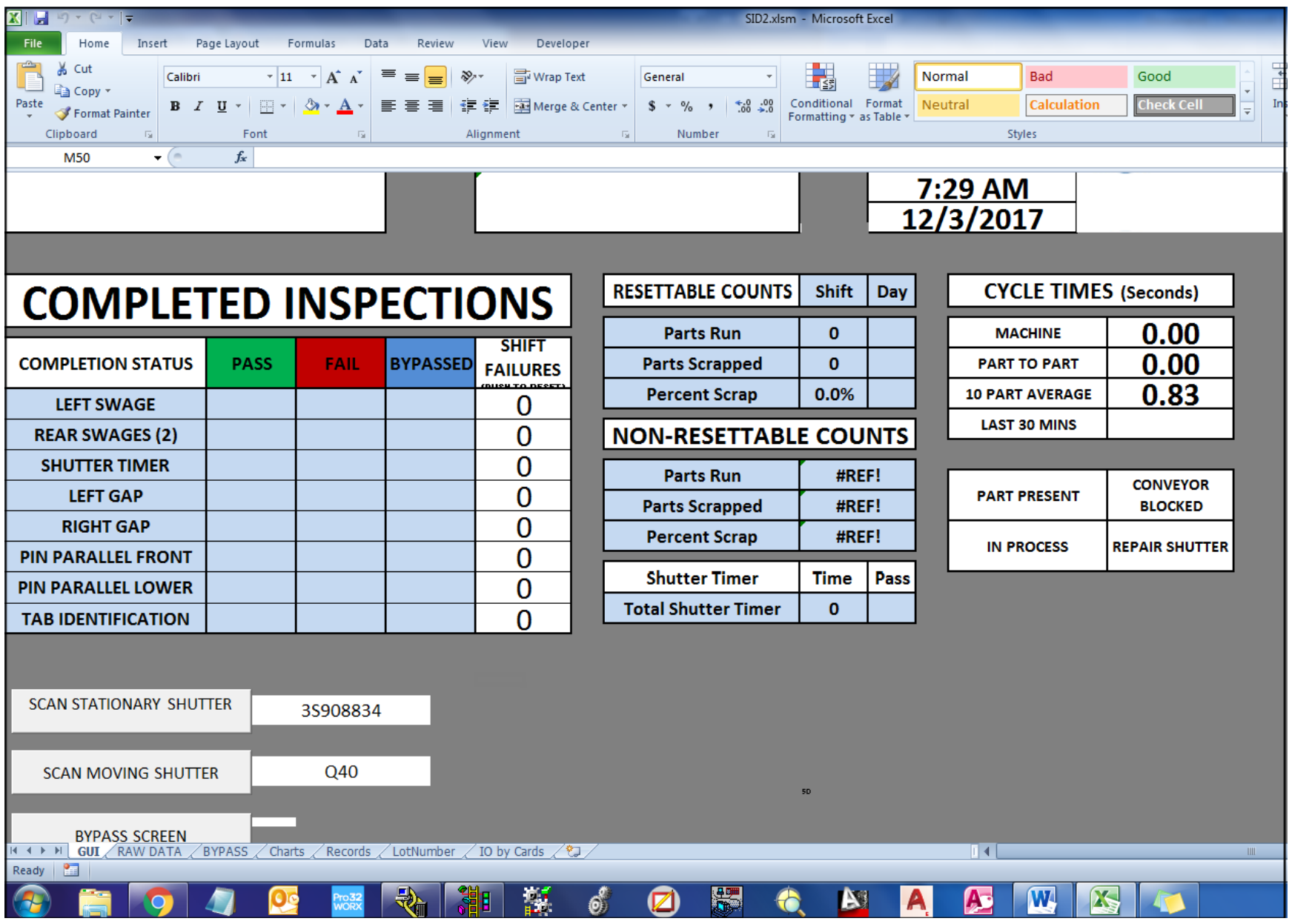

Figure 13. Excel Sheet built for Real-Time Data Reporting

\section{Summary}

Both demonstration programs and licensed programs proved reliable in their configuration, administration, and operation of data transfers from a PLC to an end-user through the data network. The work at the Industry site was an exceptional opportunity. It allowed observation of IDEC's WindO/I HMI in a real-life application. However, there was no opportunity to configure the software application. There was, however, an opportunity provided to build and test (on site) an Excel-based data monitoring display of the production line parameters. By a tag-by-tag repetition, RSLinx was used to calculate the addressing for each Excel data cell. Once the sheet was fully populated, individual cell communication via RSLinx to Excel was confirmed. However, this validation work was 
done during production line downtime. It was not possible to make use of Excel to monitor live plant production.

The licensed class laboratory programs worked well. Both Rockwell's FactoryTalk®View and Microsoft's Excel configured and operated without problems. However, there was not the opportunity to witness large-scale data transfers like those seen at the industry site. However, unlike the industry site, total control of configuration, administration, and operation was "a given" at the classroom laboratory site.

The demonstration programs procured were all tested in the laboratory. Each OPC UA application package performed without problems. The Matrikon and Kepware packages proved to have somewhat weak, or non-existent, embedded client features for use in HMI, alarming, and data collection work. Therefore the Open Automation Software client package, which had data collection and alarming features, was tested with Kepware. The Open Automation Software was used to perform data logging to a “*.csv" file and also to create "live” data within an Excel spreadsheet.

The Inductive Automation package had features not present in the Matrikon and Kepware packages. One of these features was an HMI designer, call "Ignition Designer.” The successful design created with Ignition was used as a template for the HMI design later built with FactoryTalk®View. The webbased Ignition program proved to be more "user-friendly” than the FactoryTalk®View application.

The third-party OPC UA client packages worked very well and no licensing was needed. Complete connectivity was established regardless of the chosen target OPC UA server. These results were seen as a benefit of the “open” architecture specifications established by the OPC Foundation.

The two-hour session limit for Kepware and Inductive Automation and the 30-day limit for Matrikon proved not to be an issue. Matrikon issued a reminder showing the number of days left in the trial demo. On its web-based user interface, Ignition Automation had a session countdown clock and a handy "restart session” button. Kepware also allowed for session "re-initiation.” The Open Automation Software was also time-limited to 30-days. It did not prominently display the number of days left on the temporary license.

Based on the results seen in this work, use of the OPC UA demonstration packages has a definite value. Even if the laboratory has licensed software from Rockwell and therefore RSLinx is present, there is an advantage in using the demonstration programs, as they provide the student with the opportunity to see open architecture model solutions for system-wide, PLC related, data handling.

The visit to an industry site makes it evident that PLCs and data handling are an integral part of industrial automation. Further, it is evident that industry is currently coupling software applications from different vendors to accomplish their data handling tasks. The conditions witnessed provide an argument in favor of bringing OPC UA based demonstration software products into laboratory-based and the lecture-based lessons. 


\section{References}

1. Akelian, C. J. (2015, June), Incorporating SCADA Modules into Introductory Programmable Logic Controller Curriculum Paper presented at 2015 ASEE Annual Conference \& Exposition, Seattle, Washington. 10.18260/p.24280

2. Needler, M., \& Jannotta, K. J., \& Lin, W., \& Pfile, R. (2004, June), Integration Of Enterprise And Industrial Networks In A Computer Engineering Technology Program Paper presented at 2004 Annual Conference, Salt Lake City, Utah. https://peer.asee.org/13134

3. IDEC Corporation. https://www.idec.com/

4. OPC Foundation. https://opcfoundation.org/

5. Mahnke, W., Leitner, S.H., Damm, M.. OPC Unified Architecture. Springer Science \& Business Media; 2009.

6. Gonzales, I., Calderon, A. J., Barragan, A. J., Andujar J. M., “Integration of Sensors, Controllers and Instruments Using a Novel OPC Architecture”, MDPI-Sensors, No. 17(7), 1512, DOI:10.3390/s17071512, 2017.

7. Schleipen, M., Gilani, S., Bischoff, T., Pfrommer, J., "OPC UA Industrie 4.0 - Enabling Technology with High Diversity and Variability", Procedia CIRP, vol. 57, pp. 315-320, 2016.

8. Vázquez, F.G. (2015, October), Test Platform for the Performance Evaluation of OPC-UA Servers for Fast Data Transfer Between Intelligent Equipment, Paper presented at The Fourth International Conference on Intelligent Systems and Applications (INTELLI2015), 2015, pp. 179-182, ISBN: 9781-61208-437-4. https://www.researchgate.net/

9. Imtiaz, J. and Jasperneite, J., Scalability of OPC-UA down to the chip level enables "Internet of Things", 2013 11th IEEE International Conference on Industrial Informatics (INDIN), Bochum, 2013, pp. 500-505.

10. Inductive Automation. https://inductiveautomation.com.

11. Open Automation Software. https://www.openautomationsoftware.com/why-us/why-open-automation/ 
Appendix I. Demonstration of Matrikon’s Linux-based UA Client Browser

A “prompt by prompt” report of the browsing for an OPC UA Server from the Linux command line.

Prompt blocks $1-4$ cause the browsing to advance to the OPC Server itself. Block 4 will cause the browsing to go to the Device Level. It will find the PLC device, PLC72 (see block 5).

Block 1

./DemoclientConsole_Linux64

Block 2

Discovery

Enter Discovery Url> opc.tcp://localhost:4096

Block 3

Message - User Tokens

opc.tcp: //127.0.0.1:4096/discovery

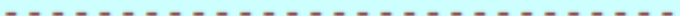

1. Username ()

Select Message Authentication (1-1) >1

Enter Username:opcuauser

Enter Password:password

Block 4

Browse

Root/

0. Objects (Objects)

1. Types (Objects)

2. Views (objects)

$[-]$ back | [x]exit

Enter selection $>0$

[objects]

(R)ead?, (B)rowse? B 
Navigation of the device level and the PLC object level.

Block 5

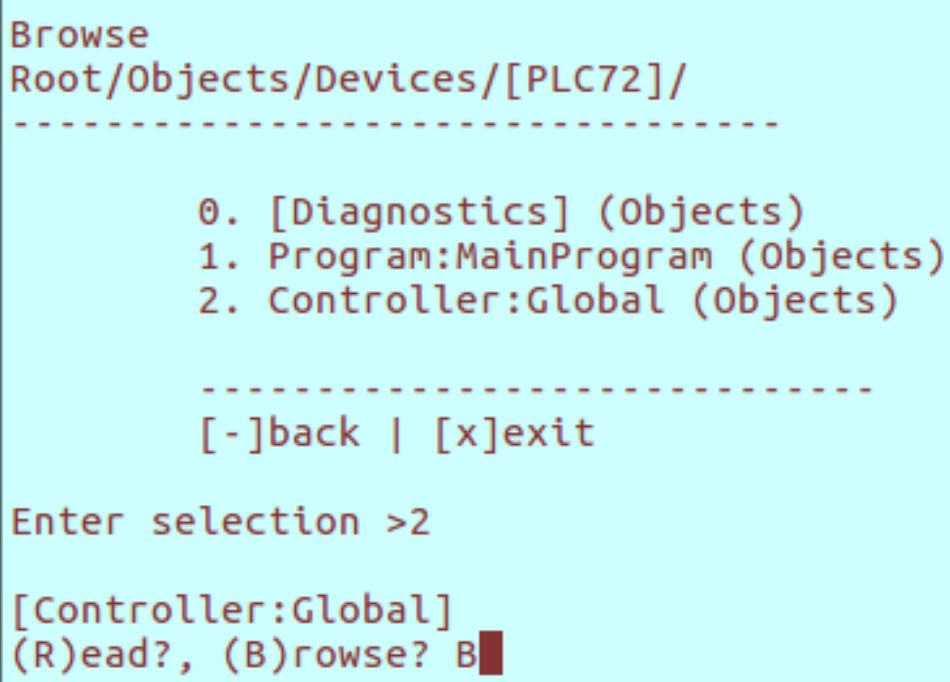

Block 6

Browse

Root/Objects/Devices/[PLC72]/Controller:Global/

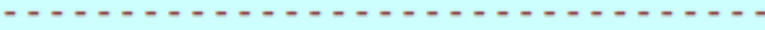

0. timer1 (objects)

1. Local:1:c (Objects)

2. stop (Variable)

3. Local:1:I (Objects)

4. t (Variable)

5. Local:1:0 (Objects)

6. stop1 (Variable)

[-]back | [x]exit

Enter selection 